\title{
Theory of error and least square adjustment: Application in coordinate transformation
}

\author{
Madhusudan Adhikari \\ President NESA
}

\begin{abstract}
After detection and elimination of blunders, determination and correction of systematic errors the remaining random errors are adjusted by the method of least square. It is very useful technique in survey adjustments; as an example of its application, adjustment of conformal transformation of coordinate has been presented in this paper.
\end{abstract}

\section{Basic concept of Errors}

Geomatics engineers are usually faced with the problem of estimating some unknown quantities (parameters). This is done through collecting several measurements of some kind known as observations- and then adopting some appropriate mathematical model relating both observations and unknowns [Naser EL-Sheimy]

Observations generally require some form of instrumentation that is operated by some observer under certain environmental condition. Therefore, in every observation these three things- instrument, observer and environment have an influence on the accuracy of the measured quantity. Due to this influence, all observations contain error - the difference between an observation of a quantity and it true value.

The true value of a quantity is never known and hence the true error too. However, both quantities can be estimated.

$$
\begin{aligned}
& \text { e }=l-t \\
& \text { e : true error } \\
& l: \text { observed value } \\
& t: \text { true value }
\end{aligned}
$$

$$
\begin{aligned}
& \mathrm{n}=l-\hat{x} \\
& \mathrm{n}: \text { estimated error } \\
& l: \text { observed value } \\
& \hat{x}: \text { estimated value }
\end{aligned}
$$

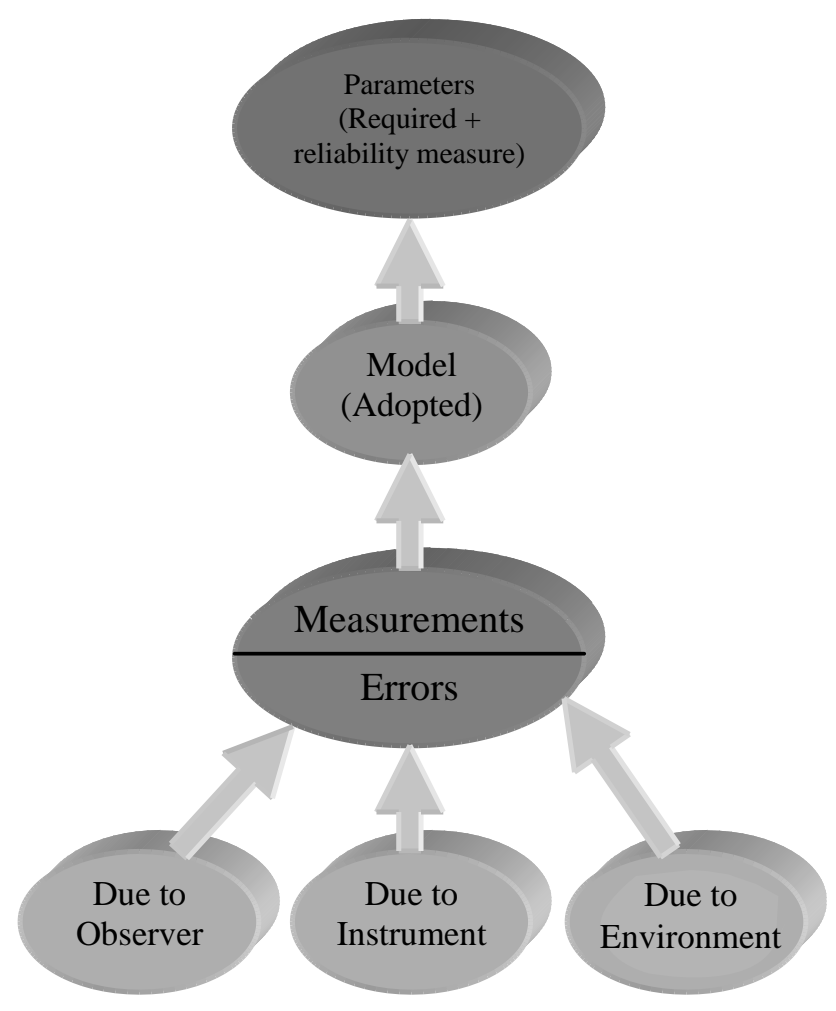




\subsection{There are three sources of error}

Personal: due to limitations in human perception power, carelessness, fatigue etc.

Instrumental: due to imperfect construction, improper and incomplete adjustment, precision limits (least count) etc.

Natural: due to changing environmental conditions, structure of the earth, gravity and gravitational forces, earth's rotation etc

\begin{tabular}{|c|c|c|c|}
\hline & Gross errors, mistakes, or blunders: & Systematic errors & Random errors \\
\hline 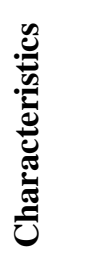 & $\begin{array}{l}\text { These are the largest of the errors likely } \\
\text { to arise/ abnormal observations }\end{array}$ & $\begin{array}{l}\text { These can be constant or variable } \\
\text { through out the observation and } \\
\text { are generally attributable to a } \\
\text { known circumstance. These can } \\
\text { be expressed by some functional } \\
\text { relationships. }\end{array}$ & $\begin{array}{l}\text { These are those variants, which } \\
\text { remain after all other errors have } \\
\text { been removed. they have no } \\
\text { functional relationship based up } \\
\text { on a deterministic system usually } \\
\text { modeled by probability theory }\end{array}$ \\
\hline ڤ્ّ & $\begin{array}{l}\text { Personal sources carelessness of the } \\
\text { observer }\end{array}$ & $\begin{array}{l}\text { All instrumental personal } \\
\text { and natural sources can cause } \\
\text { systematic error }\end{array}$ & $\begin{array}{l}\text { All instrumental personal and } \\
\text { natural sources can cause random } \\
\text { error }\end{array}$ \\
\hline 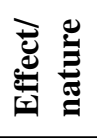 & Inhomogeneous observables & $\begin{array}{l}\text { It is cumulative in nature. It } \\
\text { shifts all the observations in a } \\
\text { certain direction. }\end{array}$ & $\begin{array}{l}\text { It is compensating in nature. } \\
\text { Cannot generally be eliminated. }\end{array}$ \\
\hline 菢 & $\begin{array}{l}\text { Must be detected by careful checking } \\
\text { and independent check measurements } \\
\text { and eliminated by filtering out }\end{array}$ & $\begin{array}{l}\text { Must be detected and corrected } \\
\text { by proper calibration of the } \\
\text { instrument before use. Use } \\
\text { appropriate procedure during } \\
\text { measurement, like reciprocal } \\
\text { observations two face reading. } \\
\text { Apply corrections. }\end{array}$ & $\begin{array}{l}\text { Can be minimized by taking } \\
\text { redundant observations and } \\
\text { adjusting by method of least } \\
\text { square. the process is referred } \\
\text { to as the "Adjustment of } \\
\text { observation" or "Adjustment } \\
\text { computation" }\end{array}$ \\
\hline
\end{tabular}

Three types of errors

\section{Principle of Least Square}

As the true value and true error by the measurements of a quantity is never known, what do we do is we estimate the most probable value (MPV) which is supposed to be the nearest to the true value among or within the range of the large no of observations. The basic theorem in this subject is referred to as Gauss-Markov theorem, which states, "in the case of independent observations of equal weight the least square estimates are the linear unbiased estimates with minimum variance." In surveying literature the principle of least square is shown derived for the observations of normal distribution and is usually expressed as- "the most probable value or the best linear unbiased estimate of a set of observations is one for which the sum of weighted squared residuals is a minimum." [w. Schofield]

The expression for the normal distribution curve, the probability curve is given by $y=\frac{h}{\sqrt{\pi}} e^{-h^{2} \mathrm{e}^{2}}$

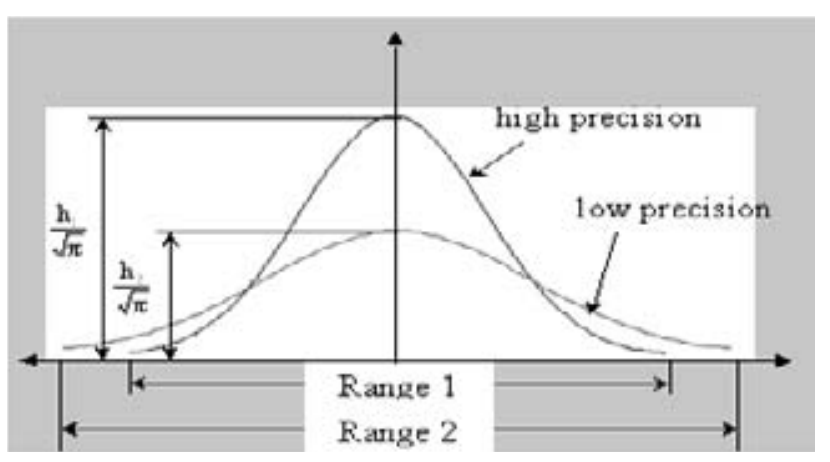

Figure 2: Probability Curve

Where

$y$-is the probability of occurrence of the error $\varepsilon$

$h$-is an index of precision

$e$-is the exponential function 2.71828 
Differentiating with respect to $h$ we will get

$$
\begin{gathered}
\frac{d y}{d h}=\frac{\mathrm{e}^{-h^{2} \varepsilon^{2}}}{\sqrt{\pi}}\left(1-2 h^{2} \varepsilon^{2}\right) \\
\text { For a maximum } y, \frac{d y}{d h}=0 \\
\text { i.e. } 1-2 h^{2} \varepsilon^{2}=0 \\
\text { or } \varepsilon^{2}=\frac{1}{2 h^{2}}
\end{gathered}
$$

Thus, the error will be decreased and the accuracy will be increased as the precision $h$ increases the maximum accuracy will be achieved when

$$
\sum_{i=1}^{n} \mathrm{e}_{i}{ }^{2}=\sum_{i=1}^{n} \frac{1}{2 h_{i}^{2}}=\mathrm{a} \text { minimum }
$$

For weighted observations

$$
\sum_{i=1}^{n} w_{i} \mathrm{e}_{i}^{2}=\mathrm{a} \text { minimum }
$$

$\varepsilon_{i}=\operatorname{MPV}(\mathrm{M})-\mathrm{i}^{\text {th }}$ observed value $\left(\mathrm{M}_{\mathrm{i}}\right)$

$w_{i}=$ weight of $\mathrm{i}^{\text {th }}$ observed value

Then,

$$
\sum_{i=1}^{n} w_{i} \mathrm{e}_{i}^{2}=\sum_{i=1}^{n} w_{i}\left(M-M_{i}\right)^{2}=\mathrm{a} \text { minimum }
$$

or $\quad \frac{d}{M}\left(\sum_{i=1}^{n} w_{i}\left(M-M_{i}\right)^{2}\right)=0$

or $\quad \sum_{i=1}^{n} w_{i}\left(M-M_{i}\right)=0$

or $\quad \sum_{i=1}^{n} w_{i}\left(M-M_{i}\right)=0$

or $\quad M \sum_{i=1}^{n} w_{i}-\sum_{i=1}^{n} w_{i} M_{i}=0$

or $\quad M=\frac{\sum_{i=1}^{n} w_{i} M_{i}}{\sum_{i=1}^{n} w_{i}}$

thus the most probable value is the weighted arithmetic mean of the observed values. if the observed values are of equal weight i.e. $w_{1}=w_{2}=w_{3}=\ldots . .=w_{n}=w$,

$$
\sum_{i=1}^{n} w_{i}=w
$$

and

$$
\sum_{i=1}^{n} w_{i} M_{i}=w \sum_{i=1}^{n} M_{i},
$$

Then $M=$

$$
\frac{\sum_{i=1}^{n} M_{i}}{n}
$$

Thus for the observations with equal weight the most probable value is the simple arithmetic mean. There are two basic methods for adjustment of the observations by this technique, namely The Indirect Method or Method of Observation Equations, and Direct Method or The Method of Condition Equations.

In the Indirect Method, we will have following four steps:

Step 1: Assume the values for required quantities (the assumed value may be the observed quantity or any other arbitrarily assumed quantity)

Step 2: Formulate the observation equations by applying corrections (vs) to the assumed values to obtain MPV.

Step 3: Determine the residuals by subtracting observed values from MPV

Step 4: formulate the normal equations by applying least square conditions.

\section{Adjustment of Conformal Transformation of Coordinates by Least Square Method}

\subsection{Conformal Transformation of Coordinates}

Let us first derive the equations for the conformal transformation. A two Dimensional Conformal Transformation consists of three basic steps:

- Scale Change

- Rotation

- Translation

\subsubsection{Scale Change}

Let us say we have coordinate systems $\mathrm{XY}$ and EN as shown in figure locating the points $a\left(X_{a}, Y_{a}\right)$ and $\mathrm{b}\left(\mathrm{X}_{\mathrm{b}}, \mathrm{Y}_{\mathrm{b}}\right)$ in $\mathrm{XY}$ system and their corresponding positions $a\left(E_{a}, N_{a}\right)$ and $b\left(E_{b}, N_{b}\right)$ in EN system, then the scale factor is given as

$$
S=A B / a b=\sqrt{\frac{\left(E_{b}-E_{a}\right)^{2}+\left(N_{b}-N_{a}\right)^{2}}{\left(X_{b}-X_{a}\right)^{2}+\left(Y_{b}-Y_{a}\right)^{2}}}
$$



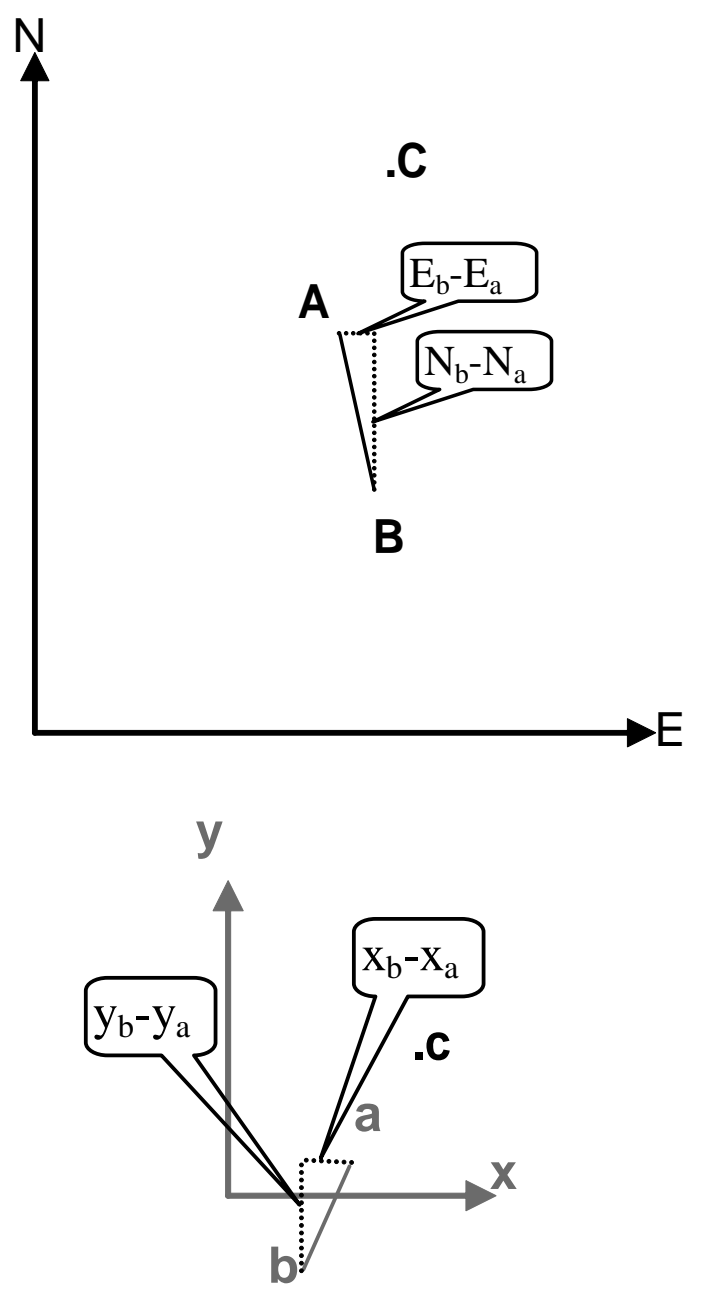

First, we will change the scale of $\mathrm{XY}$ system making equal to that of EN system by multiplying each $\mathrm{x}, \mathrm{y}$ by the scale factor $\mathrm{S}$. The scaled coordinates are $\mathrm{x}^{\prime}, \mathrm{y}^{\prime}$.

\subsubsection{Rotation}

Here we super impose the scaled x' y' coordinate system over EN system, so that $A B$ and ab of both the system coincide as shown in the figure below and construct an auxiliary axis system E'N' coinciding the origin with the origin (shifted during rotation) of x'y' system.

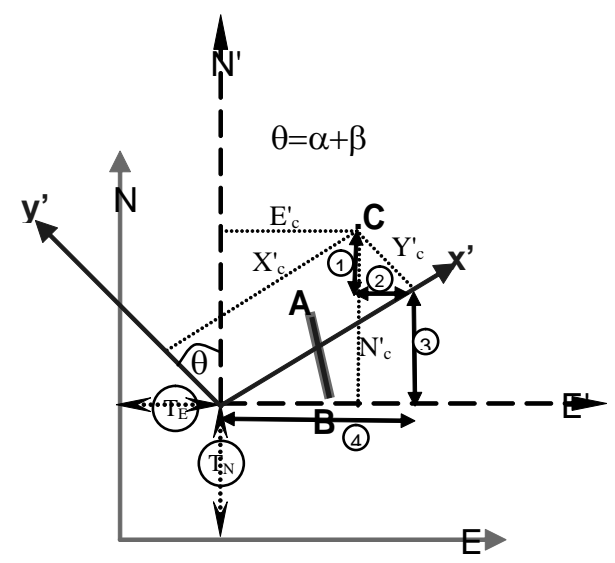

$\begin{array}{ll}\text { (1) }{ }^{y}{ }_{c}{ }_{c} \operatorname{Cos} \theta & \text { (3) }{ }^{x}{ }_{c} \operatorname{Sin} \theta \\ \text { (2) }{ }^{\prime}{ }_{c} \operatorname{Sin} \theta & \text { (4) }{ }^{x}{ }_{c}{ }_{c} \operatorname{Cos} \theta\end{array}$

$\alpha=\operatorname{Tan}^{-1}\left(\frac{\mathrm{x}_{\mathrm{b}}-\mathrm{x}_{\mathrm{a}}}{\mathrm{y}_{\mathrm{b}}-\mathrm{y}_{\mathrm{a}}}\right)$

$\beta=\operatorname{Tan}^{-1}\left(\frac{E_{B}-E_{A}}{N_{B}-N_{A}}\right)$

$\mathrm{E}^{\prime}=\mathrm{x}^{\prime} \operatorname{Cos} \theta-\mathrm{y}^{\prime} \operatorname{Sin} \theta$

$\mathrm{N}^{\prime}=\mathrm{x}^{\prime} \operatorname{Sin} \theta+\mathrm{y}^{\prime} \operatorname{Cos} \theta$

\subsubsection{Translation}

It is the Translation from E'N' coordinate system to EN system

$$
\begin{aligned}
& T_{E}=E_{A}-E_{A}^{\prime}=E_{B}-E^{\prime}{ }_{B} \\
& T_{N}=N_{A}-N_{A}^{\prime}=N_{B}-N_{B}^{\prime}
\end{aligned}
$$

Finally we determine the coordinates in EN system as

$$
\begin{aligned}
& \mathrm{E}_{\mathrm{C}}=\mathrm{T}_{\mathrm{E}}+\mathrm{E}_{\mathrm{C}}{ }_{\mathrm{C}}=\mathrm{T}_{\mathrm{E}}+\mathrm{x}^{\prime} \operatorname{Cos} \theta-\mathrm{y}^{\prime} \operatorname{Sin} \theta \\
& =\mathrm{T}_{\mathrm{E}}+\mathrm{S} \times \mathrm{x} \operatorname{Cos} \theta-\mathrm{S} \times \mathrm{y} \operatorname{Sin} \theta \\
& \mathrm{N}_{\mathrm{C}}=\mathrm{T}_{\mathrm{N}}+\mathrm{N}^{\prime}{ }_{\mathrm{C}}=\mathrm{T}_{\mathrm{N}}+\mathrm{x}^{\prime} \operatorname{Sin} \theta+\mathrm{y}^{\prime} \operatorname{Cos} \theta \\
& =\mathrm{T}_{\mathrm{N}}+\mathrm{S} \times \mathrm{x} \operatorname{Sin} \theta+\mathrm{S} \times \mathrm{y} \operatorname{Cos} \theta
\end{aligned}
$$

if we suppose $\mathrm{S} \operatorname{Sin} \theta=b$ and $\mathrm{S} \operatorname{Cos} \theta=a$ we will have the general transformation equations as

$$
\begin{aligned}
& E=T_{E}+a x-b y \\
& N=T_{N}+b x+a y
\end{aligned}
$$

\section{Transformation with Least Square Adjustment}

If more than two control points are available, redundancy exists and the transformation can be computed using least square solution.

Let $\mathrm{A}, \mathrm{B}$ and $\mathrm{C}$ are three control points known in both the $\mathrm{XY}$ and EN systems of coordinate.

As derived above, there will be 6 observation equations, two for each A, B and C, as follows: (which are with added residuals to make the redundant equations consistent.)

$$
\begin{aligned}
& a X_{A}-b Y_{A}+T_{E}=E_{A}+v_{E_{A}} \\
& a Y_{A}+b X_{A}+T_{N}=N_{A}+v_{N_{A}} \\
& a X_{B}-b Y_{B}+T_{E}=E_{B}+v_{E_{B}} \\
& a Y_{B}+b X_{B}+T_{N}=N_{B}+v_{N_{B}} \\
& a X_{C}-b Y_{C}+T_{E}=E_{C}+v_{E_{C}} \\
& a Y_{C}+b X_{C}+T_{N}=N_{C}+V_{N_{C}}
\end{aligned}
$$


For least square adjustment, let us square and add the residuals

$$
\begin{aligned}
& \sum \mathrm{v}^{2}=\left(a \mathrm{X}_{\mathrm{A}}-\mathrm{bY}_{\mathrm{A}}+\mathrm{T}_{\mathrm{E}}-\mathrm{E}_{\mathrm{A}}\right)^{2} \\
& +\left(a Y_{A}+b X_{A}+T_{N}-N_{A}\right)^{2} \\
& +\left(a X_{B}-b Y_{B}+T_{E}-E_{B}\right)^{2} \\
& +\left(a Y_{B}+b X_{B}+T_{N}-N_{B}\right)^{2} \\
& +\left(a X_{C}-b Y_{C}+T_{E}-E_{C}\right)^{2} \\
& +\left(a Y_{C}+b X_{C}+T_{N}-N_{C}\right)^{2}
\end{aligned}
$$

If we take the partial derivative of the $\Sigma \mathrm{v}^{2}$ with respect to a, b, $T_{E}$ and $T_{N}$ and compare each with zero to fulfill the condition of minima we will get four equations that can be represented by the following matrix equation.

$$
\begin{gathered}
\frac{\partial}{\partial \mathrm{a}} \sum \mathrm{v}^{2}=0, \quad \frac{\partial}{\partial \mathrm{b}} \sum \mathrm{v}^{2}=0 \\
\frac{\partial}{\partial \mathrm{T}_{\mathrm{E}}} \sum \mathrm{v}^{2}=0,
\end{gathered}
$$

Where,

$$
\begin{aligned}
& A=\left(\begin{array}{cccc}
X_{A} & -Y_{A} & 1 & 0 \\
Y_{A} & X_{A} & 0 & 1 \\
X_{B} & -Y_{B} & 1 & 0 \\
Y_{B} & X_{B} & 0 & 1 \\
X_{C} & -Y_{C} & 1 & 0 \\
Y_{C} & X_{C} & 0 & 1
\end{array}\right), \\
& X=\left(\begin{array}{c}
a \\
b \\
T_{E} \\
T_{N}
\end{array}\right), L=\left(\begin{array}{c}
E_{A} \\
N_{A} \\
E_{B} \\
N_{B} \\
E_{C} \\
N_{C}
\end{array}\right)
\end{aligned}
$$

The solution of the equation

$$
\begin{aligned}
& A^{\top} A X=A^{\top} L \text { is } \\
& X=\left(A^{\top} A\right)^{-1} A^{\top} L \\
& \text { We can get } A^{\top} A \text { from } A \text { as } \\
& \left(A^{\top} A\right)=\left(\begin{array}{cccc}
\left.\sum X_{i}^{2}+Y_{i}^{2}\right) & 0 & \sum X & \sum Y_{i} \\
0 & \left.\sum X^{2}+Y_{i}^{2}\right) & -\sum Y_{i} & \sum X_{i} \\
\sum X & -\sum Y_{i} & 3 & 0 \\
\sum Y & \sum X_{i} & 0 & 3
\end{array}\right) \\
& i=A, B, C \\
& \text { If we assume } \alpha=\sum\left(X_{i}{ }^{2}+Y_{i}{ }^{2}\right), \\
& \beta=\sum X_{i} \text { and } \gamma=\sum Y_{i}
\end{aligned}
$$

the matrix will be formed as

$$
\left(A^{\top} A\right)=\left(\begin{array}{cccc}
\alpha & 0 & \beta & \gamma \\
0 & \alpha & -\gamma & \beta \\
\beta & -\gamma & 3 & 0 \\
\gamma & \beta & 0 & 3
\end{array}\right)
$$

Calculating determinant of the matrix we will get

$$
\begin{aligned}
& \operatorname{Det}\left(A^{\top} A\right)=\psi^{2}, \\
& \text { where, } \psi=\left(3 \alpha-\beta^{2}-\gamma^{2}\right)
\end{aligned}
$$

A gain determining the inverse matrix we will get

$$
\left(A^{\top} A\right)^{-1}=\frac{1}{\psi^{2}}\left(\begin{array}{cccc}
3 \psi & 0 & -\beta \psi & -\gamma \psi \lambda \\
0 & 3 \psi & \gamma \psi & -\beta \psi \\
-\beta \psi & \gamma \psi & \alpha \psi & 0 \\
-\gamma \psi & -\beta \psi & 0 & \alpha \psi)
\end{array}\right.
$$

If we assume

$$
3 / \psi=\mathrm{p},-\beta / \psi=\mathrm{q},-\gamma / \psi=\mathrm{r}
$$

and $\alpha / \psi=\mathrm{s}$ we will have

$$
\left(A^{\top} A\right)^{-1}=\left(\begin{array}{cccc}
p & 0 & q & r \\
0 & p & -r & q \\
q & -r & s & 0 \\
r & q & 0 & s
\end{array}\right)
$$

A gain, we can get $A^{\top} L$ from $A$ and $L$ as follows

$$
A^{\top} L=\left(\begin{array}{c}
\sum\left(X_{i} E_{i}+Y_{i} N_{i}\right) \\
\sum\left(X_{i} N_{i}-Y_{i} E_{i}\right) \\
\sum_{i=A, B, C} E_{i} \\
\sum N_{i}
\end{array}\right)=\left(\begin{array}{l}
e \\
f \\
g \\
h
\end{array}\right) \text { (say) }
$$

If we operate $A^{\top} L$ with $\left(A^{\top} A\right)^{-1}$ we will get $X$

$$
X=\left(\begin{array}{c}
a \\
b \\
T_{E} \\
T_{N}
\end{array}\right)=\left(A^{\top} A^{-1} A^{\top} L=\left(\begin{array}{cccc}
p & 0 & q & r \\
0 & p & -r & q \\
q & -r & s & 0 \\
r & q & 0 & s
\end{array}\right) \times\left(\begin{array}{c}
e \\
f \\
g \\
h
\end{array}\right)\right.
$$

Finally, we will get the adjusted transformation parameters as

$$
\left(\begin{array}{c}
a \\
b \\
T_{E} \\
T_{N}
\end{array}\right)=\left(\begin{array}{c}
p e+q g+r h \\
p f-r g+q h \\
q e-r f+s g \\
r e+q f+s h
\end{array}\right)
$$


Getting these parameters, we can transform any point $\mathrm{D}$ in $\mathrm{XY}$ coordinate system to EN coordinate system as

$$
\begin{aligned}
& E_{D}=a X_{D}-b Y_{D}+T_{E} \\
& N_{D}=a Y_{D}+b X_{D}+T_{N}
\end{aligned}
$$

\section{Conclusion and Recommendations}

The theory of error and the method of Least Square adjustment has been presented in brief in this paper, the mathematical computation for the Least Square adjustment seems to be rather cumbersome if we proceed manually, it is recommended to use computer program to make its execution easier and faster. During the adjustment of survey observations or computations, one should first detect the blunders. Those observations which are larger than $3 \sigma$ i.e. E99.9 are assumed to be blunders, first they should be detected filtered out. After that the corrections to the systematic errors and biases should be applied. And finally, only the remaining random errors should be adjusted by applying the method of Least Square Adjustment.

\section{References}

1. Adhikari, $M S$, आधारभुत सर्भेक्षण

2. Arora, $K R$, Surveying vol. 2

3. Dr. Naser EL-Sheimy, Introduction to adjustment of observation

4. Schofield, W., Engineering Surveying

5. Wollf, Paul R. Elements of Photogrammetry 